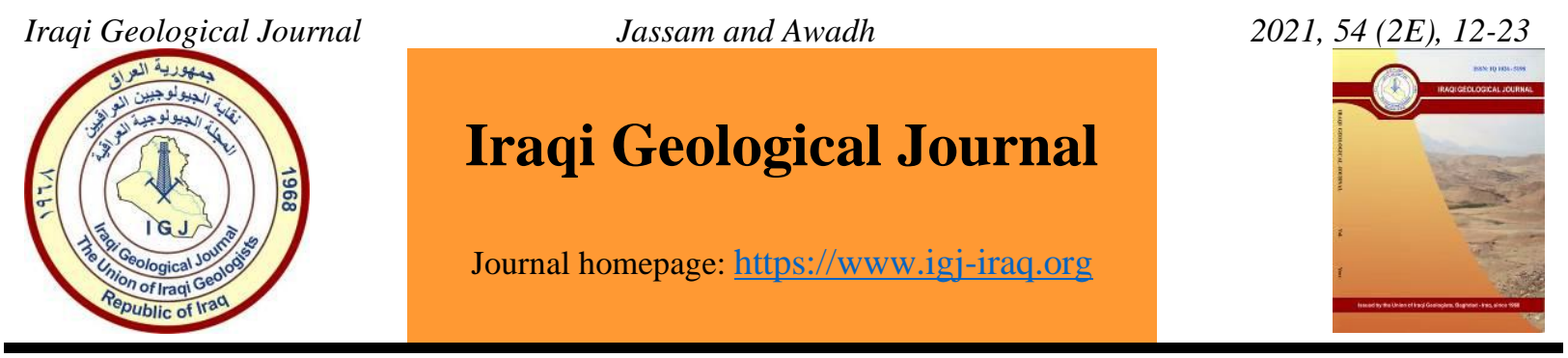

\title{
Natural Radioactivity in Soil and Bitumen in Al-Marj Spring and Abu-Jir Village, Anbar, Western Iraq
}

\author{
Haneen A. Jassam ${ }^{1}$ and Salih M. Awadh ${ }^{1, *}$ \\ 1 Department of Geology, College of Science, University of Baghdad, Baghdad, Iraq \\ * Correspondence: haneen_abd@uoanbar.edu.iq
}

Received: 16 August 2021; Accepted: 10 October 2021; Published: 30 November 2021

\begin{abstract}
The aim of the present research is to illustrate $\gamma$-ray levels emitting from soil and bitumen producing from ${ }^{238} \mathrm{U}$, ${ }^{232} \mathrm{Th}$, and ${ }^{40} \mathrm{~K}$ along Abu-Jir Fault Zone. in the area extended from Al-Marj valley to Abu-Jir village using scintillometer device. Such study is important in environmental assessment to buildup data base about radioactivity. The concentration of natural radionuclides in the study area was determined to be occurring mostly in the clay minerals and organic matter. A high purity germanium spectrometer was used to detect the activity of these elements which ranged between $100.6 \pm 18.1-1526 \pm 102,0-8.4 \pm 1.4$, and $70.1 \pm 10.9-328.2 \pm 73$ in soil, and 28.2 $\pm 5.6-94 \pm 22.1,0-2.2 \pm 0.5$, and $38.4 \pm 7.9-70.1 \pm 10.9$ in bitumen for ${ }^{226} \mathrm{Ra},{ }^{232} \mathrm{Th}$, and ${ }^{40} \mathrm{~K}$ respectively. The anomaly of the Naturally occurring radioactive material was associated with hydrocarbon accumulation and springs. The source of ${ }^{232} \mathrm{Th}$ and ${ }^{40} \mathrm{~K}$ came from the same source rock (illite), while ${ }^{226} \mathrm{Ra}$ was associated with ascending fluids. The obtained results showed that the activity of ${ }^{226} \mathrm{Ra}$ in soil has value above the world averages and other countries.
\end{abstract}

Keywords: Natural radionuclides; Bitumen; Al-Marj spring; Abu-Jir; Gamma ray

\section{Introduction}

The study area is characterized by the presence of many hydrocarbon seepages distributed along Abu-Jir Fault Zone (AJFZ), particularly, in the area extending between Hit and Abu-Jir village (Awadh et al., 2013). The outcrops of the interested area are described by rocks of Miocene age represented by carbonate facies of Euphrates Formation (Early Miocene) and evaporate facies of the Fatha Formation (Middle Miocene). Bitumen seepages are interfered and intruded with gypsum of the Fatha Formation in the area extended along Hit-Ramadi Road. At Abu-Jir village, huge deposits of bitumen ascending upward and covering the most area which may contain heavy metals and radionuclides that may be transferred to plants, thereafter may accumulate in plants and animals (Al-Rawi et al., 2014). A lot of heavy metals were found in both water and sediments of the Euphrates River in the study area (Awadh and Ahmed, 2013). Naturally occurring radioactive material (NORM) occurs everywhere and is present in the earth's crust, buildings, food, drink, and human body itself. The origin of NORM arises mainly from cosmic radiation and terrestrial radioactive material. The major source of natural radioactivity is terrestrial radiation which majority of it belong to radionuclides in ${ }^{238} \mathrm{U}$ series, ${ }^{232} \mathrm{Th}$ series, and ${ }^{40} \mathrm{~K}$, as a result of radioactive decay these elements in rocks and soil, where they can migrate in environment through soil, rocks, air, and water. These elements are present in soil in trace amounts and yields gamma

DOI: $10.46717 /$ igj.54.2E.2Ms-2021-11-18 
radiation in the environment resulting in changes in the background radiation level (Amanjeet et al., 2017). Also, they emit alpha or beta particles which may be taken into body via inhalation or ingestion. There is a direct relationship between terrestrial gamma radiation and the concentration of radionuclides in soil (Ademola et al., 2014). These radioactive elements are favorably bonded to clay minerals and organic matter $(\mathrm{OM})$ by adsorption, but in varies ability ranging from strong bonded in montmorillonite and illite to weak in kaolinite and chlorite, and less bonded to OM (Guo et al., 2021).

The problem of this study is the knowledge of the concentration of radioactive elements in the soil and bitumen and what their relationship with mineralogical content. Very limited previous studies conducted on radioactivity near the study area but limited in formation or with different medium. Some researchers studied only uranium concentration in the Euphrates Formation such Al-Bassam et al. (2006) and Al-Obaidy (2015), which attributed the enrichment of $U$ in the Euphrates Formation to ascending groundwater as a result of tectonic movement. Ali (2015) measured the activity of radioactive elements (226Ra, 232Th, 40K, and 137Cs) in sediments of the Euphrates River and springs and conclude they had high concentration in spring sediment especially 226Ra, while Farhan et al. (2020) measured radon gas $(222 \mathrm{Rn})$ concentration in the shallow groundwater of Abu-Jir village which stated the source of radon is U-rich hydrocarbons. Awadh et al. (2018) has recorded only gamma ray using the scintillometer (SSP-2-NF) device on both blocks (western and eastern) of the Abu-Jir Fault. Radioactive elements may be transferred from contaminated soil to the surface and groundwater by leaching and weathering processes, that cause deteriorates these systems if that presents at high concentrations and would become hazardous. This study aims to assess the concentration of radioactive elements $\left({ }^{238} \mathrm{U}\right.$, ${ }^{232} \mathrm{Th}$, and ${ }^{40} \mathrm{~K}$ ) in the study area to build up a database of the nature of radiation and to determine the geological materials emitted radiation.

\section{Study area}

The study area is located within the Stable Shelf in Iraq at the western side of Euphrates River. It is extended along the AJFZ from Al-Marj spring to the north, to Abu-Jir Village to the south (Fig. 1). It is restricted between latitudes $\left(33^{\circ} 15^{\prime} 33.30^{\prime \prime}-33^{\circ} 41^{\prime} 46.40^{\prime \prime} \mathrm{N}\right)$ and longitudes $\left(42^{\circ} 43^{\prime} 57.33^{\prime \prime}-42^{\circ} 54^{\prime} 11.0^{\prime \prime}\right.$ E) (Table 1). The stratigraphic succession of the area is represented by the Euphrates Formation (Early Miocene) composing of carbonates with little of clay minerals (Al-Dabbas et al., 2014 and Awadh, 2014), Fatha Formation (Middle Miocene) which is characterized by predominant evaporate facies comprised of anhydrite, gypsum, and salt, interbedded with limestone, marl, and relatively fine-grained clastics (Al-Obaidy, 2015), and Quaternary sediments which comprised from the Pleistocene and Holocene. The Pleistocene sediments are heterogeneous of fine pebbles consisting of quartz, chert, carbonate rock fragments, and clay (Awadh and Ahmed, 2013). The depositional environment of the Euphrates Formation was open to restricted platforms which indicated a lagoonal environment with warm and restricted open circulation (Al-Dabbas et al., 2013, and Al-Dabbas et al., 2014), while the depositional environment of the Fatha Formation is shallow marine water with normal to high saline water with clear oscillation in the sea level (Awadh and Al-Ankaz, 2016).

\section{Materials and Methods}

The field work was performed during 17-18 November 2020 focusing on radiometric survey to measure gamma ray using the scintillometer device (SSP-2-NF). A scale f-150 and 1500 of the scintillometers was chosen to record the whole concentrations of radionuclides ${ }^{238} \mathrm{U},{ }^{232} \mathrm{Th}$, and ${ }^{40} \mathrm{~K}$. A total of 88 measurements was measured and recorded in situ (Fig. 1). 


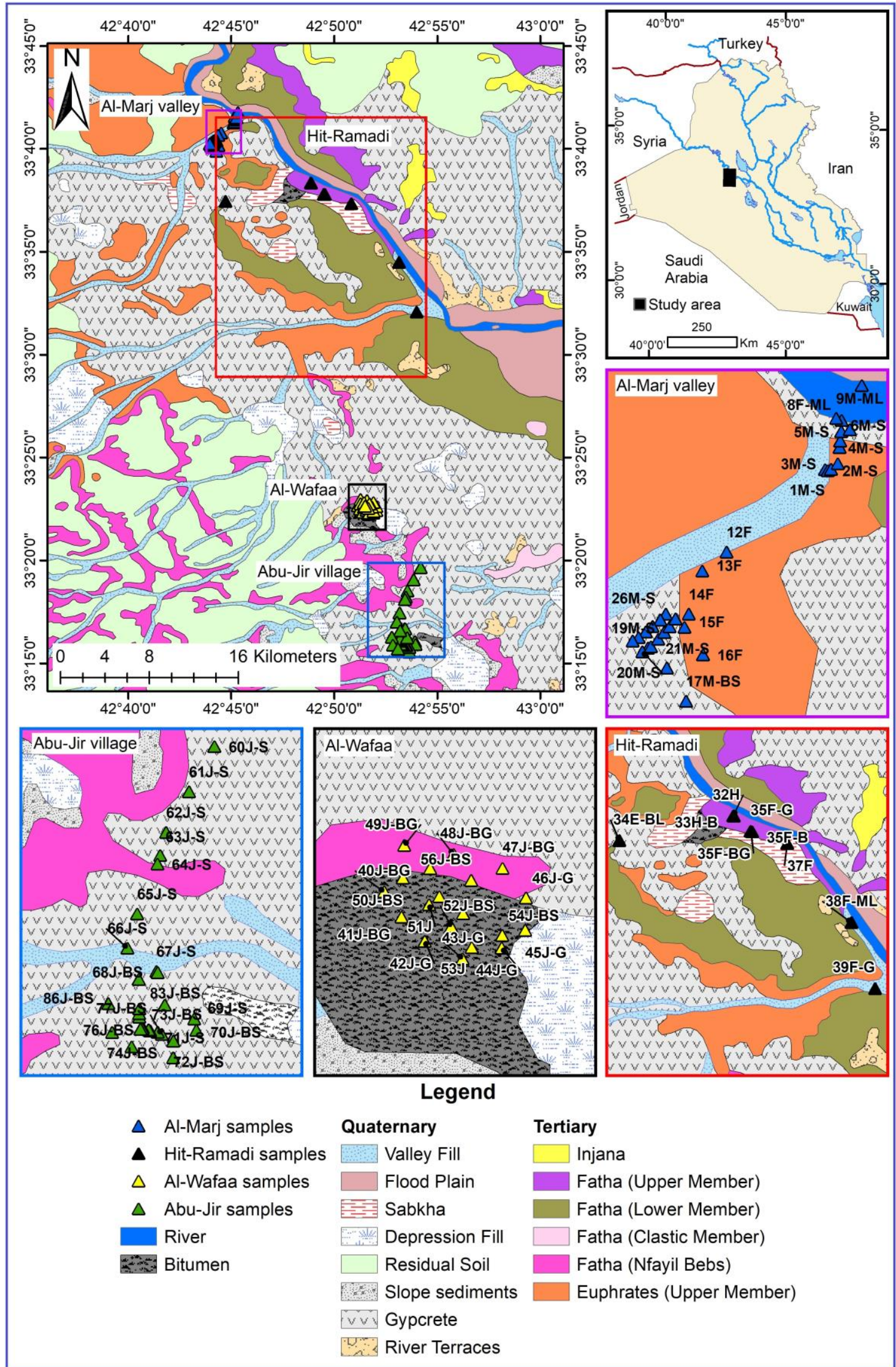

Fig.1. Geological map of the study area displays radiation reading sites along Abu-Jir Fault Zone 
Table 1. Coordinates, elevation, and samples number of the study area

\begin{tabular}{|c|c|c|c|c|c|c|c|c|c|c|c|}
\hline \multirow[t]{2}{*}{ S. No. } & \multirow[t]{2}{*}{$\begin{array}{l}\text { Ele. } \\
(\mathbf{m})\end{array}$} & \multicolumn{2}{|c|}{ Coordinates } & \multirow[t]{2}{*}{ S. No. } & \multirow[t]{2}{*}{$\begin{array}{l}\text { Ele. } \\
\text { (m) }\end{array}$} & \multicolumn{2}{|c|}{ Coordinates } & \multirow[t]{2}{*}{ S. No. } & \multirow[t]{2}{*}{$\begin{array}{l}\text { Ele. } \\
(\mathbf{m})\end{array}$} & \multicolumn{2}{|c|}{ Coordinates } \\
\hline & & Latitude & Longitude & & & Latitude & Longitude & & & Latitude & Longitude \\
\hline $1 \mathrm{M}-\mathrm{S}$ & 46.7 & $33^{\circ} 41^{\prime} 16.20^{\prime \prime}$ & $42^{\circ} 45^{\prime} 6.50^{\prime \prime}$ & $31 \mathrm{M}-\mathrm{S}$ & 81.7 & $33^{\circ} 40^{\prime} 14.50^{\prime \prime}$ & $42^{\circ} 43^{\prime} 57.33^{\prime \prime}$ & $61 \mathrm{~J}-\mathrm{S}$ & 88.7 & $33^{\circ} 19^{\prime} 3.70^{\prime \prime}$ & $42^{\circ} 53^{\prime} 51.00^{\prime \prime}$ \\
\hline $2 \mathrm{M}-\mathrm{S}$ & 47.3 & $33^{\circ} 41^{\prime} 15.80^{\prime \prime}$ & $42^{\circ} 45^{\prime} 7.40^{\prime \prime}$ & $32 \mathrm{H}$ & 75.6 & $33^{\circ} 38^{\prime} 21.51^{\prime \prime}$ & $42^{\circ} 48^{\prime} 51.20^{\prime \prime}$ & $62 \mathrm{~J}-\mathrm{S}$ & 90.2 & $33^{\circ} 18^{\prime} 31.60^{\prime \prime}$ & $42^{\circ} 53^{\prime} 33.00^{\prime \prime}$ \\
\hline $3 \mathrm{M}-\mathrm{S}$ & 48.8 & $33^{\circ} 41^{\prime} 16.30^{\prime \prime}$ & $42^{\circ} 45^{\prime} 8.60^{\prime \prime}$ & $33 \mathrm{H}-\mathrm{B}$ & 65.2 & $33^{\circ} 38^{\prime} 20.80^{\prime \prime}$ & $42^{\circ} 48^{\prime} 52.60^{\prime \prime}$ & $63 \mathrm{~J}-\mathrm{S}$ & 79.9 & $33^{\circ} 18^{\prime} 13.30^{\prime \prime}$ & $42^{\circ} 53^{\prime} 28.20^{\prime \prime}$ \\
\hline $4 \mathrm{M}-\mathrm{S}$ & 50.0 & $33^{\circ} 41^{\prime} 18.30^{\prime \prime}$ & $42^{\circ} 45^{\prime} 11.00^{\prime \prime}$ & 34E-BL & 82.6 & $33^{\circ} 37^{\prime} 27.33^{\prime \prime}$ & $42^{\circ} 44^{\prime} 43.58^{\prime \prime}$ & $64 \mathrm{~J}-\mathrm{S}$ & 91.2 & $33^{\circ} 18^{\prime} 6.50^{\prime \prime}$ & $42^{\circ} 53^{\prime} 26.00^{\prime \prime}$ \\
\hline $5 \mathrm{M}-\mathrm{S}$ & 50.0 & $33^{\circ} 41^{\prime} 24.10^{\prime \prime}$ & $42^{\circ} 45^{\prime} 11.70^{\prime \prime}$ & $35 \mathrm{~F}-\mathrm{BG}$ & 56.7 & $33^{\circ} 37^{\prime} 47.90^{\prime \prime}$ & $42^{\circ} 49^{\prime} 29.10^{\prime \prime}$ & $65 \mathrm{~J}-\mathrm{S}$ & 94.5 & $33^{\circ} 17^{\prime} 27.10^{\prime \prime}$ & $42^{\circ} 53^{\prime} 10.00^{\prime \prime}$ \\
\hline $6 \mathrm{M}-\mathrm{S}$ & 47.9 & $33^{\circ} 41^{\prime} 26.50^{\prime \prime}$ & $42^{\circ} 45^{\prime} 11.90^{\prime \prime}$ & $36 \mathrm{~F}-\mathrm{BG}$ & 74.4 & $33^{\circ} 37^{\prime} 47.18^{\prime \prime}$ & $42^{\circ} 49^{\prime} 32.31^{\prime \prime}$ & $66 \mathrm{~J}-\mathrm{S}$ & 92.9 & $33^{\circ} 17^{\prime} 0.10^{\prime \prime}$ & $42^{\circ} 53^{\prime} 2.40^{\prime \prime}$ \\
\hline $7 \mathrm{M}-\mathrm{S}$ & 51.2 & $33^{\circ} 41^{\prime} 29.90^{\prime \prime}$ & $42^{\circ} 45^{\prime} 12.30^{\prime \prime}$ & $37 \mathrm{~F}$ & 65.9 & $33^{\circ} 37^{\prime} 21.22^{\prime \prime}$ & $42^{\circ} 50^{\prime} 49.05^{\prime \prime}$ & $67 \mathrm{~J}-\mathrm{S}$ & 91.8 & $33^{\circ} 16^{\prime} 41.70^{\prime \prime}$ & $42^{\circ} 53^{\prime} 25.20^{\prime \prime}$ \\
\hline 8F-ML & 57.6 & $33^{\circ} 41^{\prime} 33.90^{\prime \prime}$ & $42^{\circ} 45^{\prime} 12.40^{\prime \prime}$ & 38F-ML & 57.9 & $33^{\circ} 34^{\prime} 29.20^{\prime \prime}$ & $42^{\circ} 53^{\prime} 08.50^{\prime \prime}$ & $68 \mathrm{~J}-\mathrm{BS}$ & 92.1 & $33^{\circ} 16^{\prime} 40.70^{\prime \prime}$ & $42^{\circ} 53^{\prime} 26.20^{\prime \prime}$ \\
\hline 9M-ML & 51.2 & $33^{\circ} 41^{\prime} 34.60^{\prime \prime}$ & $42^{\circ} 45^{\prime} 10.40^{\prime \prime}$ & $39 \mathrm{~F}-\mathrm{G}$ & 54.6 & $33^{\circ} 32^{\prime} 05.20^{\prime \prime}$ & $42^{\circ} 53 ' 59.50^{\prime \prime}$ & $69 \mathrm{~J}-\mathrm{S}$ & 86.6 & $33^{\circ} 16^{\prime} 3.90^{\prime \prime}$ & $42^{\circ} 53^{\prime} 55.20^{\prime \prime}$ \\
\hline $10 \mathrm{~F}$ & 717 & $33^{\circ} 41^{\prime} 30.58^{\prime \prime}$ & $42^{\circ} 45^{\prime} 15.41^{\prime \prime}$ & 40J-BG & 89.4 & $33^{\circ} 22^{\prime} 40.154^{\prime \prime}$ & $42^{\circ} 51^{\prime} 8.75^{\prime \prime}$ & 70J-BS & 90.2 & $33^{\circ} 15^{\prime} 55.00^{\prime \prime}$ & $42^{\circ} 53^{\prime} 56.70^{\prime \prime}$ \\
\hline $11 \mathrm{~F}$ & 65.9 & $33^{\circ} 41^{\prime} 46.40^{\prime \prime}$ & $42^{\circ} 45^{\prime} 19.62^{\prime \prime}$ & $41 \mathrm{~J}-\mathrm{BG}$ & 89.9 & $33^{\circ} 22^{\prime} 30.47^{\prime \prime}$ & $42^{\circ} 51^{\prime} 15.37^{\prime \prime}$ & $71 \mathrm{~J}-\mathrm{S}$ & 91.2 & $33^{\circ} 15^{\prime} 33.30^{\prime \prime}$ & $42^{\circ} 53^{\prime} 38.50^{\prime \prime}$ \\
\hline $12 \mathrm{~F}$ & 71.9 & $33^{\circ} 40^{\prime} 46.50^{\prime \prime}$ & $42^{\circ} 44^{\prime} 31.01^{\prime \prime}$ & $42 \mathrm{~J}-\mathrm{G}$ & 89.1 & $33^{\circ} 22^{\prime} 21.197^{\prime \prime}$ & $42^{\circ} 51 ' 24.24 "$ & 72J-BS & 92.4 & $33^{\circ} 15^{\prime} 46.40^{\prime \prime}$ & $42^{\circ} 53^{\prime} 38.70^{\prime \prime}$ \\
\hline $13 \mathrm{~F}$ & 71.0 & $33^{\circ} 40^{\prime} 39.76^{\prime \prime}$ & $42^{\circ} 44^{\prime} 22.29^{\prime \prime}$ & $43 \mathrm{~J}-\mathrm{G}$ & 86.9 & $33^{\circ} 22^{\prime} 14.571^{\prime \prime}$ & $42^{\circ} 51^{\prime} 38.63 "$ & 73J-BS & 89.6 & $33^{\circ} 15^{\prime} 51.80^{\prime \prime}$ & $42^{\circ} 53^{\prime 2} 29.60^{\prime \prime}$ \\
\hline $14 \mathrm{~F}$ & 78.1 & $33^{\circ} 40^{\prime} 24.18^{\prime \prime}$ & $42^{\circ} 44^{\prime} 17.50^{\prime \prime}$ & $44 \mathrm{~J}-\mathrm{G}$ & 86.1 & $33^{\circ} 22^{\prime} 18.64^{\prime \prime}$ & $42^{\circ} 51^{\prime} 53.51^{\prime \prime}$ & 74J-BS & 94.5 & $33^{\circ} 15^{\prime} 53.50^{\prime \prime}$ & $42^{\circ} 53^{\prime} 26.40^{\prime \prime}$ \\
\hline $15 \mathrm{~F}$ & 78.9 & $33^{\circ} 40^{\prime} 19.42^{\prime \prime}$ & $42^{\circ} 44^{\prime} 15.96^{\prime \prime}$ & $45 \mathrm{~J}-\mathrm{G}$ & 85.9 & $33^{\circ} 22^{\prime} 25.44^{\prime \prime}$ & $42^{\circ} 52^{\prime} 2.44^{\prime \prime}$ & 75J-BS & 93.9 & $33^{\circ} 15^{\prime} 54.60^{\prime \prime}$ & $42^{\circ} 53^{\prime} 20.40^{\prime \prime}$ \\
\hline $16 \mathrm{~F}$ & 76.5 & $33^{\circ} 40^{\prime} 9.57^{\prime \prime}$ & $42^{\circ} 44^{\prime} 22.58^{\prime \prime}$ & $46 \mathrm{~J}-\mathrm{G}$ & 85.9 & $33^{\circ} 22^{\prime} 37.72^{\prime \prime}$ & $42^{\circ} 52^{\prime} 2.76^{\prime \prime}$ & $76 \mathrm{~J}-\mathrm{BS}$ & 91.2 & $33^{\circ} 15^{\prime} 55.50^{\prime \prime}$ & $42^{\circ} 53^{\prime} 19.10^{\prime \prime}$ \\
\hline $17 \mathrm{M}-\mathrm{BS}$ & 79.3 & $33^{\circ} 39^{\prime} 52.94^{\prime \prime}$ & $42^{\circ} 44^{\prime} 16.53^{\prime \prime}$ & 47J-BG & 86.5 & $33^{\circ} 22^{\prime} 49.085^{\prime \prime}$ & $42^{\circ} 51$ '53.74" & 77J-BS & 94.8 & $33^{\circ} 15^{\prime} 56.80^{\prime \prime}$ & $42^{\circ} 53^{\prime} 14.60^{\prime \prime}$ \\
\hline $18 \mathrm{M}-\mathrm{S}$ & 76.3 & $33^{\circ} 40^{\prime} 4.86^{\prime \prime}$ & $42^{\circ} 44^{\prime} 9.66^{\prime \prime}$ & $48 \mathrm{~J}-\mathrm{BG}$ & 86.7 & $33^{\circ} 22^{\prime} 54.46^{\prime \prime}$ & $42^{\circ} 51^{\prime} 35.165^{\prime \prime}$ & $78 \mathrm{~J}-\mathrm{BS}$ & 95.1 & $33^{\circ} 15^{\prime} 56.20^{\prime \prime}$ & $42^{\circ} 53^{\prime} 14.10^{\prime \prime}$ \\
\hline $19 \mathrm{M}-\mathrm{S}$ & 74.1 & $33^{\circ} 40^{\prime} 11.97^{\prime \prime}$ & $42^{\circ} 44^{\prime} 2.83^{\prime \prime}$ & $49 \mathrm{~J}-\mathrm{BG}$ & 86.9 & $33^{\circ} 22^{\prime} 57.78^{\prime \prime}$ & $42^{\circ} 51^{\prime} 16.409^{\prime \prime}$ & 79J-BS & 96.7 & $33^{\circ} 15^{\prime} 56.30^{\prime \prime}$ & $42^{\circ} 53^{\prime} 13.90^{\prime \prime}$ \\
\hline $20 \mathrm{M}-\mathrm{S}$ & 75.2 & $33^{\circ} 40^{\prime} 10.60^{\prime \prime}$ & $42^{\circ} 44^{\prime} 0.81^{\prime \prime}$ & $50 \mathrm{~J}-\mathrm{BS}$ & 88.9 & $33^{\circ} 22^{\prime} 45.33^{\prime \prime}$ & $42^{\circ} 51^{\prime} 15.86^{\prime \prime}$ & 80J-BS & 93.6 & $33^{\circ} 15^{\prime} 55.80^{\prime \prime}$ & $42^{\circ} 53^{\prime} 12.60^{\prime \prime}$ \\
\hline $21 \mathrm{M}-\mathrm{S}$ & 82.3 & $33^{\circ} 40^{\prime} 12.45^{\prime \prime}$ & $42^{\circ} 44^{\prime} 3.95^{\prime \prime}$ & $51 \mathrm{~J}$ & 88.9 & $33^{\circ} 22^{\prime} 35.23^{\prime \prime}$ & $42^{\circ} 51^{\prime} 25.873^{\prime \prime}$ & 81J-BS & 96.9 & $33^{\circ} 16^{\prime} 5.50^{\prime \prime}$ & $42^{\circ} 53^{\prime} 11.70^{\prime \prime}$ \\
\hline $22 \mathrm{M}-\mathrm{S}$ & 87.2 & $33^{\circ} 40^{\prime} 15.15^{\prime \prime}$ & $42^{\circ} 44^{\prime} 6.52^{\prime \prime}$ & $52 \mathrm{~J}-\mathrm{BS}$ & 87.3 & $33^{\circ} 22^{\prime} 26.824^{\prime \prime}$ & $42^{\circ} 51^{\prime} 34.305^{\prime \prime}$ & 82J-BS & 96.7 & $33^{\circ} 16^{\prime} 8.30^{\prime \prime}$ & $42^{\circ} 53^{\prime} 11.50^{\prime \prime}$ \\
\hline $23 \mathrm{M}-\mathrm{S}$ & 75.0 & $33^{\circ} 40^{\prime} 17.60^{\prime \prime}$ & $42^{\circ} 44^{\prime} 8.70^{\prime \prime}$ & $53 \mathrm{~J}$ & 85.7 & $33^{\circ} 22^{\prime} 18.816^{\prime \prime}$ & $42^{\circ} 51^{\prime} 42.027^{\prime \prime}$ & $83 \mathrm{~J}-\mathrm{BS}$ & 98.2 & $33^{\circ} 16^{\prime} 12.00^{\prime \prime}$ & $42^{\circ} 53^{\prime} 11.90^{\prime \prime}$ \\
\hline $24 \mathrm{M}-\mathrm{S}$ & 85.1 & $33^{\circ} 40^{\prime} 19.68^{\prime \prime}$ & $42^{\circ} 44^{\prime} 10.59^{\prime \prime}$ & $54 \mathrm{~J}-\mathrm{BS}$ & 85.9 & $33^{\circ} 22^{\prime} 23.473^{\prime \prime}$ & $42^{\circ} 51^{\prime} 53.715^{\prime \prime}$ & $84 \mathrm{~J}-\mathrm{BS}$ & 93.2 & $33^{\circ} 16^{\prime} 14.98^{\prime \prime}$ & $42^{\circ} 53^{\prime} 31.92^{\prime \prime}$ \\
\hline $25 \mathrm{M}-\mathrm{S}$ & 83.2 & $33^{\circ} 40^{\prime} 22.47^{\prime \prime}$ & $42^{\circ} 44^{\prime} 12.73^{\prime \prime}$ & $55 \mathrm{~J}-\mathrm{BS}$ & 85.2 & $33^{\circ} 22^{\prime} 35.831^{\prime \prime}$ & $42^{\circ} 51^{\prime} 48.912^{\prime \prime}$ & $85 \mathrm{~J}-\mathrm{BS}$ & 95.5 & $33^{\circ} 16^{\prime} 35.30^{\prime \prime}$ & $42^{\circ} 53^{\prime} 11.33^{\prime \prime}$ \\
\hline $26 \mathrm{M}-\mathrm{S}$ & 74.4 & $33^{\circ} 40^{\prime} 24.26^{\prime \prime}$ & $42^{\circ} 44^{\prime} 9.23^{\prime \prime}$ & $56 \mathrm{~J}-\mathrm{BS}$ & 86.2 & $33^{\circ} 22^{\prime} 44.44^{\prime \prime}$ & $42^{\circ} 51^{\prime} 41.861^{\prime \prime}$ & $86 \mathrm{~J}-\mathrm{BS}$ & 100.2 & $33^{\circ} 16^{\prime} 16.07^{\prime \prime}$ & $42^{\circ} 52^{\prime} 47.37^{\prime \prime}$ \\
\hline $27 \mathrm{M}-\mathrm{S}$ & 74.7 & $33^{\circ} 40^{\prime} 22.11^{\prime \prime}$ & $42^{\circ} 44^{\prime} 7.27^{\prime \prime}$ & $57 \mathrm{~J}-\mathrm{BS}$ & 87.4 & $33^{\circ} 22^{\prime} 48.925^{\prime \prime}$ & $42^{\circ} 51^{\prime} 26.214^{\prime \prime}$ & $87 \mathrm{~J}-\mathrm{B}$ & 99.7 & $33^{\circ} 15^{\prime} 53.26^{\prime \prime}$ & $42^{\circ} 52^{\prime} 50.28^{\prime \prime}$ \\
\hline $28 \mathrm{M}-\mathrm{S}$ & 78.7 & $33^{\circ} 40^{\prime} 19.79^{\prime \prime}$ & $42^{\circ} 44^{\prime} 4.49^{\prime \prime}$ & $58 \mathrm{~J}-\mathrm{BS}$ & 86.9 & $33^{\circ} 22^{\prime} 31.843^{\prime \prime}$ & $42^{\circ} 51^{\prime} 38.78^{\prime \prime}$ & 88J-BS & 98.7 & $33^{\circ} 15^{\prime} 42.06^{\prime \prime}$ & $42^{\circ} 53^{\prime} 5.58^{\prime \prime}$ \\
\hline 29M-S & 80.8 & $33^{\circ} 40^{\prime} 17.80^{\prime \prime}$ & $42^{\circ} 44^{\prime} 2.02^{\prime \prime}$ & 59J-BS & 86.9 & $33^{\circ} 22^{\prime} 38.287^{\prime \prime}$ & $42^{\circ} 51 ' 29.74 "$ & & & & \\
\hline 30M-S & 82.6 & $33^{\circ} 40^{\prime} 15.77^{\prime \prime}$ & $42^{\circ} 43^{\prime} 59.44^{\prime \prime}$ & $60 \mathrm{~J}-\mathrm{S}$ & 93.9 & $33^{\circ} 19^{\prime} 39.50^{\prime \prime}$ & $42^{\circ} 54^{\prime} 11.00^{\prime \prime}$ & & & & \\
\hline
\end{tabular}

M: Al-Marj valley; S: soil; F: Fatha Fm.; ML: marly limestone; BS: bituminous soil; H: Hit; B: bitumen; E: Euphrates Fm.; BL: bituminous limestone; BG: bituminous gypsum; G: gypsum; J: Abu-Jir village.

The Surfer 13 program was used to design isorad map which display the variation in the radioactivity. Sixteen samples were collected from the Quaternary sediments, Fatha and Euphrates formations. Soil samples were collected at a depth of $10 \mathrm{~cm}$, while samples from formations were collected randomly from the accessible areas. Samples (4M-S, 5M-S, 35F-B, 35F-BG, 39F-G, 68J-BS, 70J-BS, 82J-BS, 87J-B) were subjected to mineralogical identification using X-ray diffraction (XRD), geochemical analysis by inductively coupled plasma mass spectrometry (ICP-MS), and radioactivity detection via high purity germanium (HPGe) detector. Samples were prepared in the Geochemistry Laboratory at the Department of Geology, College of Science, University of Baghdad. Each sample was dried at room temperature and was crashed into fine powder using porcelain mortar and Fritsch pulverisette (Buhrke et al., 1998). XRD was carried out in Directorate of Materials Research, Ministry 
of Science and Technology. Mineralogical composition was detected by semi-quantitative estimations, while the mineral abundance was evaluated according to normative calculation. Geochemical analysis was performed in the Mineral Division -ALS Chemex, Spain (Sevilla). The radioactive analysis was conducted in the Radiation Protection Center, Ministry of Health and Environment on $1 \mathrm{~kg}$ weight of sample which was preserved in sealed bags for four weeks to achieve secular equilibrium between ${ }^{226} \mathrm{Ra}$, ${ }^{232} \mathrm{Th}$, and their progenies (Veiga et al., 2006).

\section{Results}

The results are presented in Table 2 . The field measuring is represented by the field recording of the scintillometer (SSP-2-NF) for gamma ray. The data modified after calibration of the device with 137Cs to obtain the calibration constant, which equal to 3.24 , thereafter, the field results multiplied by calibration constant and 3 or 30 for $150 / \mathrm{F}$ and 1500 scale of scintillometer respectively. The field measurements presented in Table 2 were plotted in an isorad map by Surfer 13 program which displays the distribution of radioactivity in the study area (Fig. 2).

Table 2. Gamma ray based on the scintillometer readings in the field

\begin{tabular}{|c|c|c|c|c|c|}
\hline S. No. & $\gamma$-ray $(\mathrm{c} / \mathrm{s})$ & S. No. & $\gamma$-ray $(\mathrm{c} / \mathrm{s})$ & S. No. & $\gamma$-ray $(\mathrm{c} / \mathrm{s})$ \\
\hline $1 \mathrm{M}-\mathrm{S}$ & 194.4 & $31 \mathrm{M}-\mathrm{S}$ & 388.8 & $61 \mathrm{~J}-\mathrm{S}$ & 82.62 \\
\hline $2 \mathrm{M}-\mathrm{S}$ & 174.96 & $32 \mathrm{H}$ & 136.08 & $62 \mathrm{~J}-\mathrm{S}$ & 77.76 \\
\hline $3 M-S$ & 145.8 & $33 \mathrm{H}-\mathrm{B}$ & 106.92 & $63 \mathrm{~J}-\mathrm{S}$ & 106.92 \\
\hline $4 \mathrm{M}-\mathrm{S}$ & 145.8 & $34 \mathrm{E}-\mathrm{BL}$ & 136.08 & $64 \mathrm{~J}-\mathrm{S}$ & 87.48 \\
\hline $5 \mathrm{M}-\mathrm{S}$ & 194.4 & $35 \mathrm{~F}-\mathrm{BG}$ & 68.04 & $65 \mathrm{~J}-\mathrm{S}$ & 77.76 \\
\hline $6 \mathrm{M}-\mathrm{S}$ & 194.4 & $36 \mathrm{~F}-\mathrm{BG}$ & 126.36 & $66 \mathrm{~J}-\mathrm{S}$ & 77.76 \\
\hline $7 \mathrm{M}-\mathrm{S}$ & 174.96 & $37 \mathrm{~F}$ & 116.64 & $67 \mathrm{~J}-\mathrm{S}$ & 87.48 \\
\hline 8F-ML & 97.2 & 38F-ML & 131.22 & $68 \mathrm{~J}-\mathrm{BS}$ & 145.8 \\
\hline 9M-ML & 116.64 & $39 \mathrm{~F}-\mathrm{G}$ & 87.48 & $69 \mathrm{~J}-\mathrm{S}$ & 87.48 \\
\hline $10 \mathrm{~F}$ & 126.36 & $40 \mathrm{~J}-\mathrm{BG}$ & 213.84 & $70 \mathrm{~J}-\mathrm{BS}$ & 243 \\
\hline $11 \mathrm{~F}$ & 155.52 & $41 \mathrm{~J}-\mathrm{BG}$ & 174.96 & $71 \mathrm{~J}-\mathrm{S}$ & 106.92 \\
\hline $12 \mathrm{~F}$ & 136.08 & $42 \mathrm{~J}-\mathrm{G}$ & 165.24 & 72J-BS & 87.48 \\
\hline $13 \mathrm{~F}$ & 140.94 & $43 \mathrm{~J}-\mathrm{G}$ & 165.24 & $73 \mathrm{~J}-\mathrm{BS}$ & 165.24 \\
\hline $14 \mathrm{~F}$ & 136.08 & $44 \mathrm{~J}-\mathrm{G}$ & 155.52 & $74 \mathrm{~J}-\mathrm{BS}$ & 194.4 \\
\hline $15 \mathrm{~F}$ & 145.8 & $45 \mathrm{~J}-\mathrm{G}$ & 174.96 & $75 \mathrm{~J}-\mathrm{BS}$ & 204.12 \\
\hline $16 \mathrm{~F}$ & 126.36 & $46 \mathrm{~J}-\mathrm{G}$ & 165.24 & $76 \mathrm{~J}-\mathrm{BS}$ & 145.8 \\
\hline $17 \mathrm{M}-\mathrm{BS}$ & 155.52 & $47 \mathrm{~J}-\mathrm{BG}$ & 174.96 & $77 \mathrm{~J}-\mathrm{BS}$ & 243 \\
\hline $18 \mathrm{M}-\mathrm{S}$ & $340.2^{*}$ & $48 \mathrm{~J}-\mathrm{BG}$ & 194.4 & $78 \mathrm{~J}-\mathrm{BS}$ & 174.96 \\
\hline $19 \mathrm{M}-\mathrm{S}$ & $1069^{*}$ & $49 \mathrm{~J}-\mathrm{BG}$ & 204.12 & $79 \mathrm{~J}-\mathrm{BS}$ & 243 \\
\hline $20 \mathrm{M}-\mathrm{S}$ & $1166^{*}$ & $50 \mathrm{~J}-\mathrm{BS}$ & 398.52 & $80 \mathrm{~J}-\mathrm{BS}$ & 174.96 \\
\hline $21 \mathrm{M}-\mathrm{S}$ & $826.2^{*}$ & $51 \mathrm{~J}$ & 320.76 & $81 \mathrm{~J}-\mathrm{BS}$ & 174.96 \\
\hline $22 \mathrm{M}-\mathrm{S}$ & $583.2^{*}$ & $52 \mathrm{~J}-\mathrm{BS}$ & 427.68 & $82 \mathrm{~J}-\mathrm{BS}$ & 262.44 \\
\hline $23 \mathrm{M}-\mathrm{S}$ & $486^{*}$ & $53 \mathrm{~J}$ & 340.2 & $83 \mathrm{~J}-\mathrm{BS}$ & 194.4 \\
\hline $24 \mathrm{M}-\mathrm{S}$ & $476.3^{*}$ & $54 \mathrm{~J}-\mathrm{BS}$ & 379.08 & $84 \mathrm{~J}-\mathrm{BS}$ & 213.84 \\
\hline $25 \mathrm{M}-\mathrm{S}$ & $243^{*}$ & $55 \mathrm{~J}-\mathrm{BS}$ & 583.2 & $85 \mathrm{~J}-\mathrm{BS}$ & 194.4 \\
\hline $26 \mathrm{M}-\mathrm{S}$ & 349.92 & $56 \mathrm{~J}-\mathrm{BS}$ & 330.48 & $86 \mathrm{~J}-\mathrm{BS}$ & 145.8 \\
\hline $27 \mathrm{M}-\mathrm{S}$ & 155.52 & $57 \mathrm{~J}-\mathrm{BS}$ & 903.96 & $87 \mathrm{~J}-\mathrm{B}$ & 97.2 \\
\hline $28 \mathrm{M}-\mathrm{S}$ & 243 & $58 \mathrm{~J}-\mathrm{BS}$ & 534.6 & $88 \mathrm{~J}-\mathrm{BS}$ & 194.4 \\
\hline $29 \mathrm{M}-\mathrm{S}$ & 252.72 & $59 \mathrm{~J}-\mathrm{BS}$ & 583.2 & & \\
\hline $30 \mathrm{M}-\mathrm{S}$ & 272.16 & $60 \mathrm{~J}-\mathrm{S}$ & 77.76 & & \\
\hline
\end{tabular}




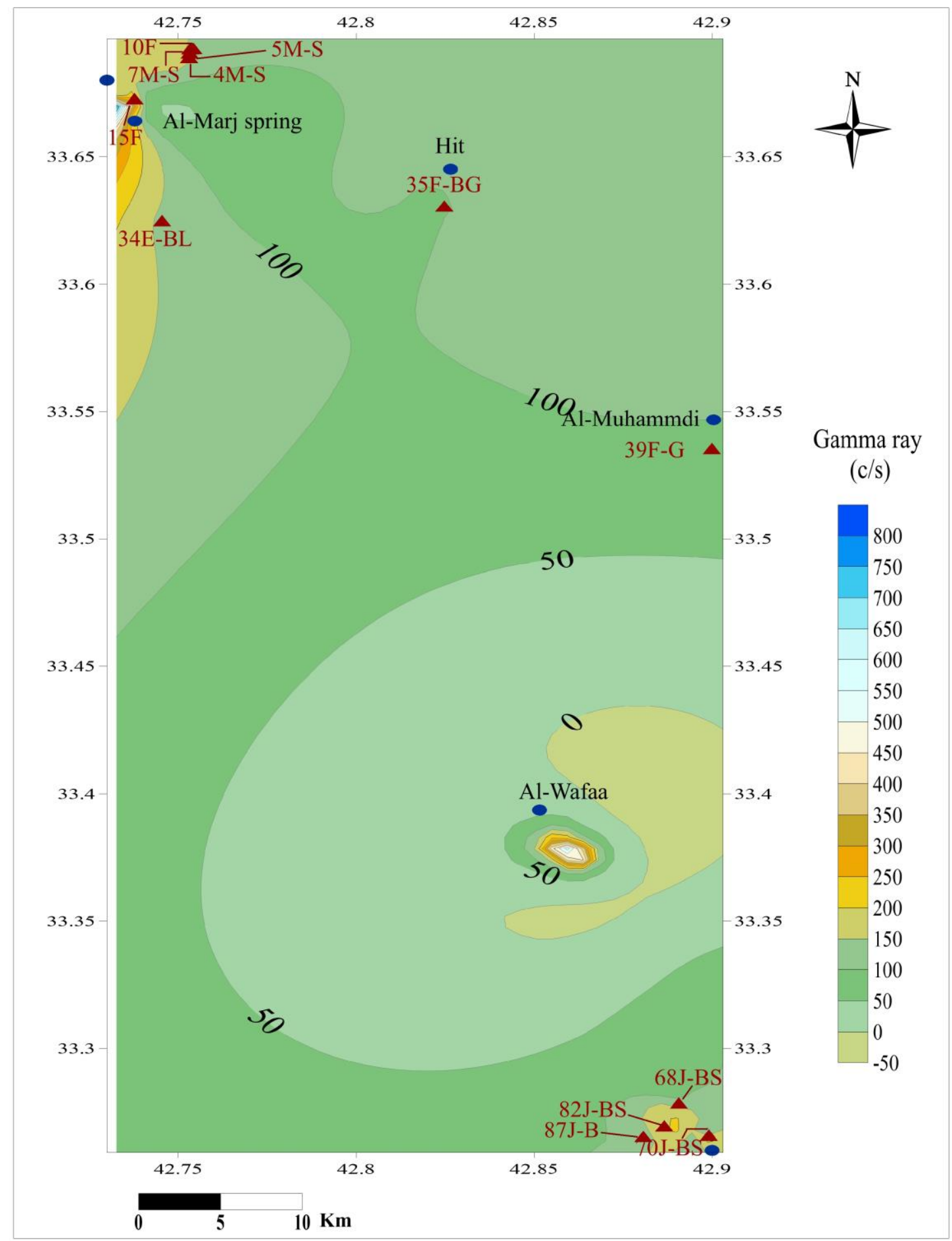

Fig. 2. Isorad map displays the radioactivity in the study area

The semi quantitative estimation for XRD analysis of soil samples showed a prevalence calcite mineral, followed by dolomite, then quartz and clay minerals. The normative calculation of mineral composition is listed in Table 3 based on chemical analysis of major and minor oxides $(\mathrm{CaO}, \mathrm{MgO}$, $\mathrm{SiO}_{2}, \mathrm{Al}_{2} \mathrm{O}_{3}, \mathrm{Fe}_{2} \mathrm{O}_{3}, \mathrm{Na}_{2} \mathrm{O}, \mathrm{K}_{2} \mathrm{O}, \mathrm{TiO}_{2}, \mathrm{MnO}, \mathrm{P}_{2} \mathrm{O}_{5}, \mathrm{SO}_{3}$, and $\mathrm{LOI}$ ) as well as the concentration of $\mathrm{U}$ and Th in ppm as listed in (Table 4). 
Table 3. Mineralogical composition of samples collected from Al-Marj valley and Abu-Jir village

\begin{tabular}{|c|c|c|c|c|c|c|c|c|c|c|}
\hline \multirow[t]{2}{*}{ S. No. } & \multirow[t]{2}{*}{ Formation } & \multicolumn{5}{|c|}{ Mineralogical composition (\%) } & \multicolumn{3}{|c|}{$\mathbf{C M}$} & \multirow[t]{2}{*}{ Total } \\
\hline & & Calcite & Dolomite & Gypsum & Quartz & $\mathbf{C M}^{*}$ & $\mathbf{I}^{*}$ & $\mathbf{K}^{*}$ & $\mathbf{P}^{*}$ & \\
\hline $4 \mathrm{M}-\mathrm{S}$ & Quaternary & 22.08 & 24.76 & 2.69 & 8.38 & 35.17 & 18.6 & 11.0 & 5.6 & 93.08 \\
\hline $5 \mathrm{M}-\mathrm{S}$ & sediment & 57.75 & 28.09 & & 10.0 & 4.25 & 2.5 & 1.2 & 0.7 & 100.09 \\
\hline $35 \mathrm{~F}-\mathrm{BG}$ & Fatha & & & 13.7 & & & & & & 13.7 \\
\hline $39 \mathrm{~F}-\mathrm{G}$ & Formation & & & 93.01 & & & & & & 93.01 \\
\hline $68 \mathrm{~J}-\mathrm{BS}$ & & 33.58 & 2.91 & & 5.01 & 12.24 & 5.6 & 1.8 & 4.8 & 53.74 \\
\hline $70 \mathrm{~J}-\mathrm{BS}$ & Quaternary & 35.04 & 8.12 & & 5.28 & 20.94 & 8.4 & 3.7 & 8.9 & 69.38 \\
\hline $82 \mathrm{~J}-\mathrm{BS}$ & & 38.37 & & & 6.92 & 14.69 & 6.3 & 1.4 & 6.96 & 59.98 \\
\hline Min & & 22.08 & 2.91 & & 5.01 & 4.25 & & & & \\
\hline Max & & 57.75 & 28.09 & & 10 & 35.17 & & & & \\
\hline Av. & & 37.36 & 15.97 & & 7.12 & 17.46 & & & & \\
\hline
\end{tabular}

${ }^{*}$ CM: Clay minerals; I: Illite; K: Kaolinite; P: Palygorskite

Table 4. Results of major, minor oxides, and traces of samples in the study area

\begin{tabular}{lccccccccccccccc}
\hline S. No. & $\mathbf{C a O}$ & $\mathbf{M g O}$ & $\mathbf{S i O}_{2}$ & $\mathrm{Al}_{2} \mathbf{O}_{3}$ & $\mathbf{F e}_{2} \mathbf{O}_{3}$ & $\mathbf{N a}_{2} \mathbf{O}$ & $\mathbf{K}_{2} \mathbf{O}$ & $\mathbf{T i O}_{2}$ & $\mathbf{M n O}$ & $\mathbf{P}_{2} \mathbf{O}_{5}$ & $\mathbf{S O}_{3}$ & $\mathbf{L O I}^{*}$ & Total & $\mathbf{U}$ & $\mathbf{T h}$ \\
\hline & & & & & & & & $\mathbf{\%}$ & & & & & & & ppm \\
4M-S & 19.9 & 6.81 & 26.8 & 7.86 & 4.09 & 2.18 & 1.35 & 0.42 & 0.06 & 0.19 & 1.25 & 30.2 & 101.21 & 2.3 & 4.64 \\
5M-S & 40.9 & 6.32 & 12.3 & 0.89 & 0.51 & 0.09 & 0.18 & 0.05 & 0.01 & 0.05 & 0.25 & 38.9 & 100.55 & 2.27 & 0.78 \\
39F-G & 33.3 & 0.49 & 0.6 & 0.13 & 0.06 & 0.03 & 0.05 & 0.01 & $<0.01$ & 0.01 & 43.3 & 21.5 & 99.47 & 0.2 & 0.09 \\
68J-BS & 19.7 & 1.52 & 11.7 & 1.96 & 0.5 & 0.74 & 0.41 & 0.14 & 0.02 & 0.06 & 13.8 & 53.2 & 103.8 & 0.65 & 1.47 \\
70J-BS & 22.1 & 3.34 & 16.7 & 3.43 & 1.5 & 0.28 & 0.61 & 0.21 & 0.04 & 0.1 & 7.25 & 45.8 & 101.5 & 1.18 & 2.11 \\
82J-BS & 21.5 & 1.35 & 15.1 & 2.06 & 0.75 & 1.36 & 0.46 & 0.16 & 0.01 & 0.06 & 10.3 & 50.5 & 103.7 & 0.62 & 1.49 \\
Min & 19.7 & 0.49 & 0.6 & 0.13 & 0.06 & 0.03 & 0.05 & 0.01 & $<0.01$ & 0.01 & 7.25 & 21.5 & - & 0.2 & 0.09 \\
Max & 40.9 & 6.81 & 26.8 & 7.86 & 4.09 & 2.18 & 1.35 & 0.42 & 0.06 & 0.19 & 43.3 & 53.2 & - & 2.3 & 4.64 \\
Av. & 26.2 & 3.31 & 13.9 & 2.72 & 1.24 & 0.78 & 0.51 & 0.17 & 0.03 & 0.08 & 18.6 & 40 & - & 1.2 & 1.76 \\
\hline
\end{tabular}

*loss on ignition

The radioactivity levels of ${ }^{226} \mathrm{Ra},{ }^{232} \mathrm{Th}$, and ${ }^{40} \mathrm{~K}$ were measured in the samples that collected from the study area and the results in $\mathrm{Bq} / \mathrm{kg}$ are presented in Table 5 and Fig.3. The radionuclide of ${ }^{238} \mathrm{U}$-series yields ${ }^{226} \mathrm{Ra}$ isotope, so it can be represented as a reference alternative of ${ }^{238} \mathrm{U}$.

Table 5. The specific activity of ${ }^{226} \mathrm{Ra},{ }^{232} \mathrm{Th}$, and ${ }^{40} \mathrm{~K}$ in $\mathrm{Bq} / \mathrm{kg}$ based on HPGe

\begin{tabular}{lccc}
\hline \multirow{2}{*}{ S. No. } & \multicolumn{3}{c}{ Specific activity $(\mathbf{B q} / \mathbf{k g})$} \\
\cline { 2 - 4 } & ${ }^{226} \mathrm{Ra}$ & ${ }^{232} \mathrm{Th}$ & ${ }^{40} \mathrm{~K}$ \\
$4 \mathrm{M}-\mathrm{S}$ & $100.6 \pm 18.1$ & $8.4 \pm 1.4$ & $328.2 \pm 73$ \\
$5 \mathrm{M}-\mathrm{S}$ & $474.4 \pm 95$ & & $127.8 \pm 23.1$ \\
35F-B & $28.2 \pm 5.6$ & $2.2 \pm 0.5$ & $38.4 \pm 7.9$ \\
35F-BG & $12.8 \pm 2.4$ & $2.9 \pm 0.1$ & $35.8 \pm 2.4$ \\
39F-G & $34.6 \pm 4.3$ & $3.4 \pm 2$ & $39.8 \pm 5$ \\
68J-BS & $236 \pm 15$ & $2.2 \pm 0.7$ & $130 \pm 14$ \\
70J-BS & $1526 \pm 102$ & $7.6 \pm 1.2$ & $298 \pm 14$ \\
82J-BS & $668 \pm 19.3$ & $4.4 \pm 0.5$ & $281 \pm 13.4$ \\
87J-B & $94 \pm 22.1$ & & $70.1 \pm 10.9$ \\
Av. & $352.73 \pm 31.53$ & $4.44 \pm 0.91$ & $149.9 \pm 18.19$ \\
\hline
\end{tabular}

\section{Discussion}

The specific activity of ${ }^{226} \mathrm{Ra},{ }^{232} \mathrm{Th}$, and ${ }^{40} \mathrm{~K}$ in the soil samples were found in the range of $100.6 \pm 18.1$ to $1526 \pm 102,0$ to $8.4 \pm 1.4$, and $127.8 \pm 23.1$ to $328.2 \pm 73$ respectively. There is a large variation in the concentration of ${ }^{226} \mathrm{Ra}$, especially in bituminous soil (BS) samples as shown in Fig. 3. 


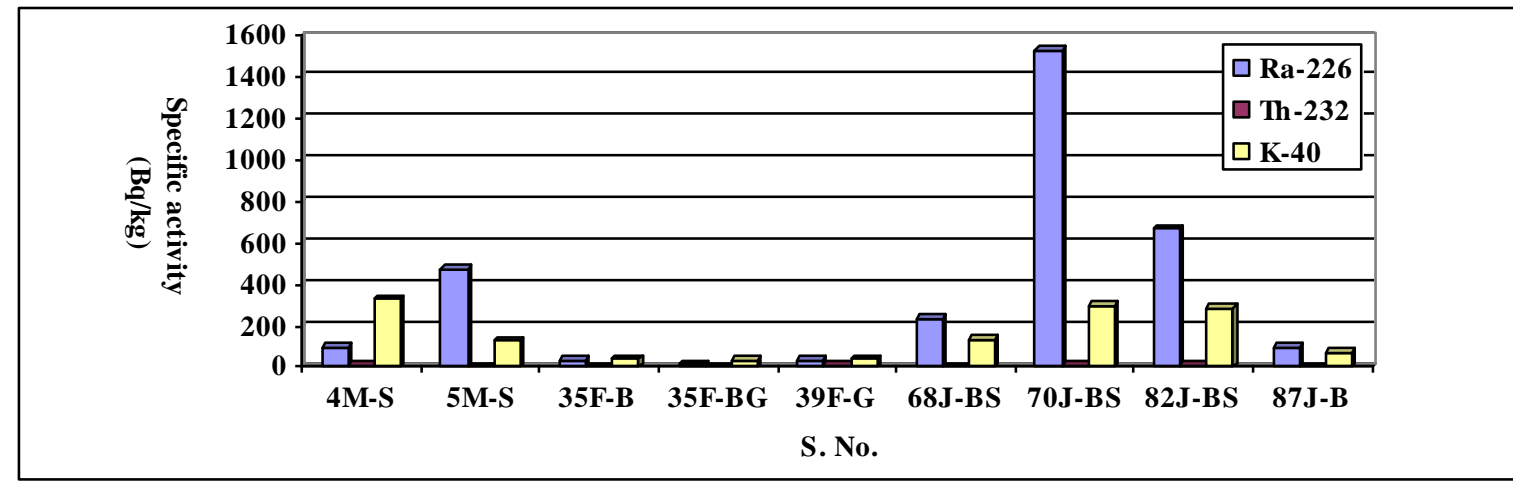

Fig.3. The specific activity of ${ }^{226} \mathrm{Ra},{ }^{232} \mathrm{Th}$ and ${ }^{40} \mathrm{~K}$ in the study area

This might be due to variation in mineralogical content in soil which may be attributed mainly to clay minerals and organic matter constitutes. The clay minerals provide a favorable surface to attach radioactive elements by adsorption, but the organic matter is least able to attach these elements. Native bitumen has no capacity for attractive uranium as appearing in 35F-B and 87J-B samples (Table 5), but some of the organic acids that are consist of very minor contents of petroliferous matters may readily make uranium salts (Bell, 1963) which may interpret the high activity of radium in bituminous soil samples (70J-BS and 82J-BS) in Table 4. Mahdi and Al-Kadhimi (2007) attribute the radioactive anomaly in the study area to their association with spring and hydrocarbon deposits which spread along AJFZ in the area, as well as, the outcrops of the Euphrates Formation that proved to contain unordinary concentration of uranium in the upper part of the formation (Al-Atia and Mehdi, 2005). The location of hydrocarbon seepages was closely to the samples collected from Abu-Jir village that also explain the high activity of these samples and the higher value of gamma radiation in the samples from Al-Marj spring (from 18M-S to 26M-S readings), as well as, the seepages close to Al-Wafaa area (from 50J-BS to 59J-BS readings) as shown in Table 2 . In 5M-S sample, the radium activity may attribute to carbonate content which has fossiliferous constitutes that adsorb radioactive elements.

The concentration of uranium in soil samples in ppm does not cooperate with their specific activity of radium, that was belonged to circumstances of the host rocks. This can illustrate by the influence the oxidation state which yields to chelate of uranium thereafter establish disruption of radioactive equilibrium of U-series then tendency in favor of radium (Al-Atia and Mehdi, 2005). The specific activity of ${ }^{40} \mathrm{~K}$ is attributed to clay minerals content in samples, especially illite which has a strong ability to adsorption with radioactive elements (Guo et al., 2021), that is reflected in the activity of ${ }^{40} \mathrm{~K}$ of sample (4M-S) which associated with high clay mineral abundance comparing with other samples, this is show obviously in Table 3 and Table 5.

The correlation coefficient was done between the specific activity of ${ }^{226} \mathrm{Ra},{ }^{232} \mathrm{Th}$, and ${ }^{40} \mathrm{~K}$ which appeared that they all have positive correlation with each other as shown in Fig. $4 .{ }^{232} \mathrm{Th}$ has a good positive correlation with ${ }^{40} \mathrm{~K}(\mathrm{r}=0.89)$, that be owing to the fact the radionuclides develop from the same rock formation (Munyao et al., 2020), which is illite due to potassium in its structure as the main constitute. While ${ }^{226} \mathrm{Ra}$ has a poor positive correlation with ${ }^{232} \mathrm{Th}$ and ${ }^{40} \mathrm{~K}$, that means the source of ${ }^{226} \mathrm{Ra}$ from ascending fluids as a result of its high mobility, that transport ${ }^{226} \mathrm{Ra}$ from source rock and adsorption on clay minerals and organic matter. This is explained that the variation occurring between ${ }^{226} \mathrm{Ra}$ with ${ }^{232} \mathrm{Th}$ and ${ }^{40} \mathrm{~K}$ in different samples. Finally, by comparison of the average of specific activity of ${ }^{226} \mathrm{Ra},{ }^{232} \mathrm{Th}$, and ${ }^{40} \mathrm{~K}$ in soil samples with their activity of other countries in Table 6 , that reveals the average concentration of ${ }^{226} \mathrm{Ra}$ in the study area is higher than other countries and the world average, hydrocarbon leakages are consider the source of uranium (radium) in the area (Farhan et al., 2020). The concentration of ${ }^{232} \mathrm{Th}$ and ${ }^{40} \mathrm{~K}$ is lower than the world average value and other countries except for Jordan and Saudi Arabia (Asir) have a lower concentration of ${ }^{40} \mathrm{~K}$ than the present study. The variation 
in the concentration of radionuclides in the soil of various regions of the world depends on the geological and geographical condition of each region of the world, as well as, using chemical fertilizers which applied to agricultural lands in some regions (El-Taher and Al-Zahrani, 2014) which provide radionuclides and heavy metals to soil, especially ${ }^{40} \mathrm{~K}$.
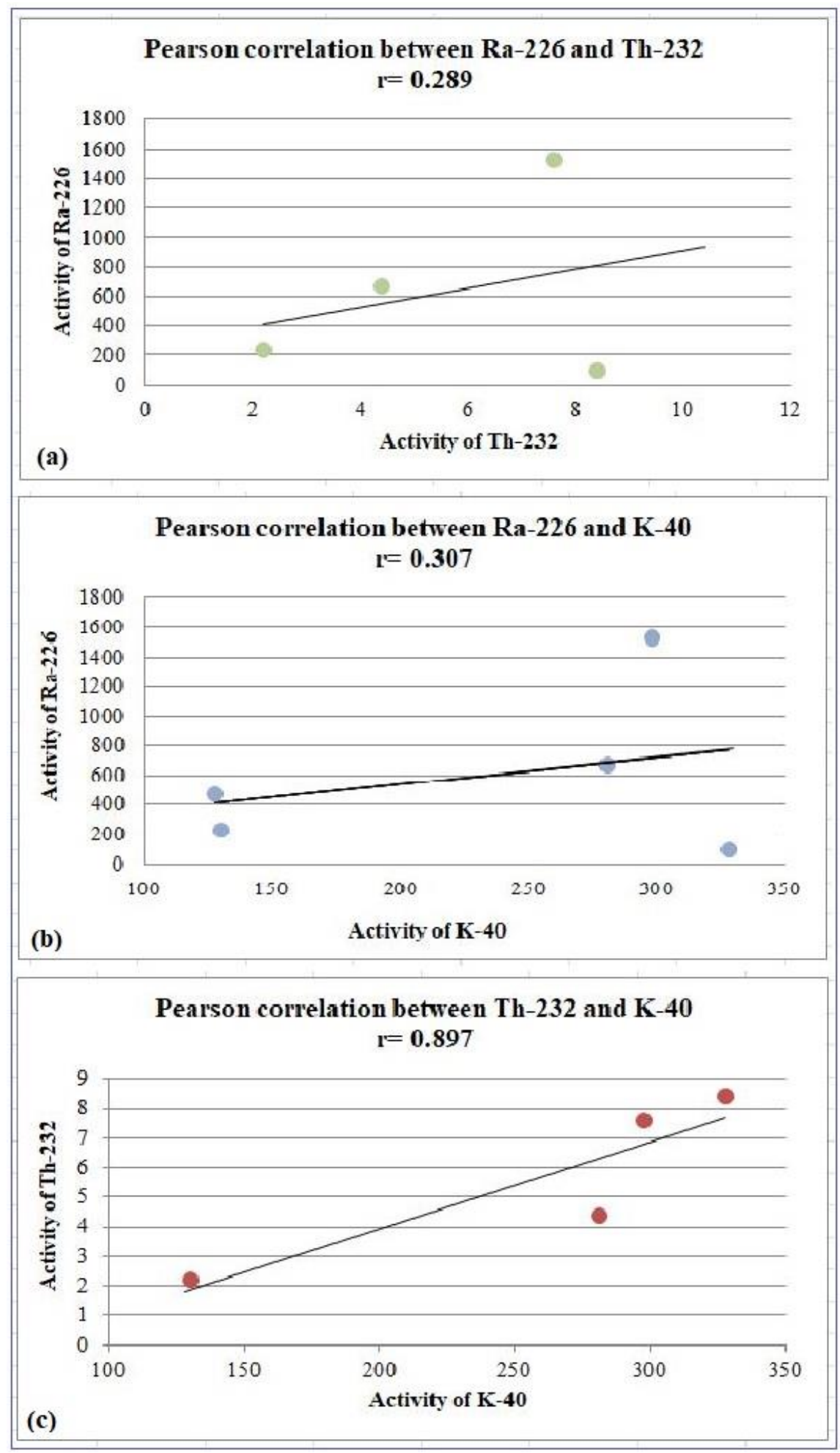

Fig.4. Person correlation plot showing the correlation between: (a) ${ }^{226} \mathrm{Ra}$ and ${ }^{232} \mathrm{Th}$; (b) ${ }^{226} \mathrm{Ra}$ and ${ }^{40} \mathrm{~K}$; (c) ${ }^{232} \mathrm{Th}$ and ${ }^{40} \mathrm{~K}$. 
Table 6. The specific activity of ${ }^{226} \mathrm{Ra},{ }^{232} \mathrm{Th}$, and ${ }^{40} \mathrm{~K}$ in $\mathrm{Bq} / \mathrm{kg}$ in soil samples in different countries and the present study

\begin{tabular}{|c|c|c|c|c|}
\hline \multirow{2}{*}{ Country } & \multicolumn{3}{|c|}{ Specific activity (Bq/kg) } & \multirow{2}{*}{ References } \\
\hline & ${ }^{226} \mathbf{R a}$ & ${ }^{232} \mathbf{T h}$ & ${ }^{40} \mathbf{K}$ & \\
\hline Iraq (Nineveh) & 33.55 & 21.52 & 326.74 & (Najam and Younis, 2015) \\
\hline Northern India & 63.88 & 91.56 & 340.78 & (Mehra and Singh, 2012) \\
\hline Turkey & 32 & 22.86 & 1318 & (Aközcan et al., 2014) \\
\hline Iraq (Baghdad-Karakh side) & 16.47 & 9.72 & 367.95 & (Abojassim and Rasheed, 2019) \\
\hline Jordan & 44.9 & 18.1 & 138.4 & (Saleh and Shayeb, 2014) \\
\hline Yemen & 30.41 & 36.26 & 358.12 & (Harb et al., 2014) \\
\hline Egypt & 17 & 18 & 320 & (UNSCEAR, 2000) \\
\hline Malaysia & 67 & 82 & 310 & (UNSCEAR, 2000) \\
\hline Iran & 38.8 & 43.4 & 555.1 & (Asgharizadeh et al., 2013) \\
\hline Egypt (Abou Zabal region) & 31.12 & 10.96 & 264.1 & (Diab et al., 2008) \\
\hline Saudi Arabia (Asir) & 38.7 & 23.5 & 217.9 & (Ibraheem et al., 2018) \\
\hline Algeria & 53.2 & 50.03 & 311 & (Boukhenfouf and Boucenna, 2011) \\
\hline Brazil (Panama) & 10.22 & 7.27 & 54.75 & (Becegato et al., 2008) \\
\hline Saudi Arabia (Qassim) & 9.5 & 12.3 & 535 & (El-Taher and Al-Zahrani, 2014) \\
\hline World average & 33 & 45 & 420 & (UNSCEAR, 2014) \\
\hline Iraq (Al-Marj spring to Abu-Jir village) & 601 & 5.65 & 233 & The present study \\
\hline
\end{tabular}

\section{Conclusions}

The presence the study area along Abu-Jir Fault Zone is a matter of worth, especially many phenomena are associated with it like springs and hydrocarbon seepages. This study is proved the presence of radiation along this fault which cannot separate the origin of this radiation from the phenomena related. The variation is obviously in soil samples and became more obvious in bituminous soil samples of Abu-Jir village, which due to clay minerals and organic materials constitutes in samples. This variation is appeared in ${ }^{226} \mathrm{Ra}$ activity which increases in bituminous soil, while bitumen samples have lower activity than soil, as a result of high mobility of radium from rich-uranium hydrocarbon seeps. The correlation analysis proved the positive correlation among radioactive elements which mean that elements came from the same source. The comparison of the activity of naturally occurring radioactive elements with other countries showed the study area has higher concentration of ${ }^{226} \mathrm{Ra}$ than over the world averages and other countries.

\section{Acknowledgements}

The authors gratefully acknowledge Mr. Laith A. Al-Hity for accommodating us during the field work. The authors thank the Department of Geology, University of Baghdad, for providing the Laboratory of Geochemistry and Scintillation Counter. The authors are very grateful to the Secretary of Journal Mr. Samir R. Hijab. and the Technical Editors for their great efforts and valuable comments.

\section{References}

Abojassim, A. A., \& Rasheed, L. H., 2019. Mapping of terrestrial gamma radiation in soil samples at Baghdad Governorate (Karakh Side), using GIS technology. Nature Environment and Pollution Technology, 18(4), 1095-1106.

Ademola, A. K., Bello, A. K., \& Adejumobi, A. C., 2014. Determination of natural radioactivity and hazard in soil samples in and around gold mining area in Itagunmodi, south-western, Nigeria. Journal of Radiation research and applied sciences, 7(3), 249-255.

Aközcan, S., Yılmaz, M., \& Külahcı, F., 2014. Dose rates and seasonal variations of 238 U, 232 Th, 226 Ra 40 K and 137 Cs radionuclides in soils along Thrace, Turkey. Journal of Radioanalytical and Nuclear Chemistry, 299(1), 95-101. 
Al-Atia, M. J., \& Mehdi, M. A., 2005. Origin and mode of formation of Abu Skhair uranium deposits, middle Iraq. Iraqi Bulletin of Geology and Mining (Arabic), 1(1), 15-27.

Al-Bassam, K. S., Mahdi, M. A., \& Al-Delaimi, M. R., 2006. Contribution to the origin of the syngenetic uranium enrichment in the Early Miocene carbonates of the Euphrates Formation, Iraq. Iraqi Bulletin of Geology and Mining, 2(2), 1-21.

Al-Dabbas, M. A., Awadh, S. M., \& Zaid, A. A., 2014. Facies analysis and geochemistry of the Euphrates Formation in Central Iraq. Arabian Journal of Geosciences, 7(5), 1799-1810.

Al-Dabbas, M., Awadh, S.M. \& Zaid, A.A., 2013. Mineralogy, geochemistry, and reserve estimation of the Euphrates limestone for Portland cement industry at Al-Najaf area, South Iraq. Arabian Journal of Geosciences, 6(2), 491-503.

Ali, K. K., 2015. Radionuclides content and the radiological hazard of sediment of the Euphrates River and sprigs-western Iraq. Iraqi Journal of Science, 56(2A), 1098-1110.

Al-Obaidy, S. A. H., 2015. Geochemical Survey of the Oligocene - Miocene Exposures and Recent Soil between Al - Qaim and Ramadi, West of Iraq. Ph. D. Thesis, University of Baghdad. 238.

Al-Rawi, F.R., Al-Ameri, T.K., \& Awadh, S.M., 2014. Petroleum geochemistry of oil samples from shallow boreholes at Sakran site, western Iraq. Arabian Journal of Geosciences, 7(2), 545-558.

Amanjeet, Kumar, A., Kumar, S., Singh, J., Singh, P., \& Bajwa, B. S., 2017. Assessment of natural radioactivity levels and associated dose rates in soil samples from historical city Panipat, India. Journal of radiation research and applied sciences, 10(3), 283-288.

Asgharizadeh, F., Ghannadi, M., Samani, A. B., Meftahi, M., Shalibayk, M., Sahafipour, S. A., \& Gooya, E. S., 2013. Natural radioactivity in surface soil samples from dwelling areas in Tehran city, Iran. Radiation protection dosimetry, 156(3), 376-382.

Awadh, S. M., \& Ahmed, R. M., 2013, Hydrochemistry and pollution probability of selected sites along the Euphrates River, Western Iraq. Arabian Journal of Geosciences, 6(7), 2501-2518.

Awadh, S. M., Ali, K. K., \& Alazzawi, A. T., 2013. Geochemical exploration using surveys of spring water, hydrocarbon and gas seepage, and geobotany for determining the surface extension of Abu-Jir Fault Zone in Iraq: A new way for determining geometrical shapes of computational simulation models. Journal of Geochemical Exploration, 124, 218-229.

Awadh, S. M., 2014. Stable carbon and nitrogen isotopes and elemental composition and origin of organic matter from the Neogene Euphrates, Injana and Dibdibba formations in Iraq: discrimination between marine and terrestrial environments. Geological Quarterly, 58, 729-736.

Awadh, S. M., \& Al-Ankaz, Z. S., 2016. Sulfur isotopes geochemistry of the bituminous Fatha evaporates in Fatha Formation, Hit-Abu Jir area, western Iraq. Iraqi Geological Journal, 34-45.

Awadh, S. M., Al-Bahadily, H. A., \& Al-Ankaz, Z. S., 2018. Interpreting the tectonics of the Abu Jir fault, Karbla-Najaf plateau using mineralogical and geophysical data. Iraqi Bulletin of Geology and Mining, 14(1), 47-63.

Becegato, V. A., Ferreira, F. J. F., \& Machado, W. C. P., 2008. Concentration of radioactive elements (U, Th and K) derived from phosphatic fertilizers in cultivated soils. Brazilian Archives of Biology and Technology, 51(6), 1255-1266.

Bell, K. G., 1963. Uranium in carbonate rocks, 474-A.

Boukhenfouf, W., \& Boucenna, A., 2011. The radioactivity measurements in soils and fertilizers using gamma spectrometry technique. Journal of environmental radioactivity, 102(4), 336-339.

Buhrke, V. E., Jenkins, R., Smith, D. K., \& Kingsley, D., 1998. Practical guide for the preparation of specimens for $\mathrm{X}$-ray fluorescence and $\mathrm{x}$-ray diffraction analysis. Wiley-VCH.

Diab, H. M., Nouh, S. A., Hamdy, A., \& El-Fiki, S. A., 2008. Evaluation of natural radioactivity in a cultivated area around a fertilizer factory. Nuclear and Radiation Physics, 3(1), 53-62.

El-Taher, A., \& Al-Zahrani, J. H., 2014. Radioactivity measurements and radiation dose assessments in soil of Al-Qassim region, Saudi Arabia.

Farhan, A. O., Ahmed, A. M., Awadh, S. M., \& Al-Sulttani, A. H., 2020. Radon gas and effective dose in groundwater in Abu-Jir Village in Anbar, Western Iraq. Iraqi Geological Journal, 26-33.

Guo, W., Chen, G., Li, Y., Li, Y., Zhang, Y., Zhou, J., ... \& Dang, H., 2021. Factors controlling the Lower radioactivity and its relation with higher organic matter content for Middle Jurassic Oil Shale in Yuqia 
Depression, Northern Qaidam Basin, China: evidence from organic and inorganic geochemistry. ACS omega, 6(11), 7360-7373.

Harb, S., El-Kamel, A. H., Zahran, A. M., Abbady, A., \& Ahmed, F. A., 2014. Assessment of natural radioactivity in soil and water samples from Aden governorate south of Yemen region. International Journal of Recent Research in Physics and Chemical Sciences, 1(1), 1-7.

Ibraheem, A. A., El-Taher, A., \& Alruwaili, M. H., 2018. Assessment of natural radioactivity levels and radiation hazard indices for soil samples from Abha, Saudi Arabia. Results in Physics, 11, 325-330.

Mahdi, M. A., \& Al-Kadhimi, J. A., 2007. Origin of radiometric anamonlies deduced from airborne geophysical surveys in Iraq (Arabic). Iraqi Bulletin of Geology and Mining, 3(1), 1-11.

Mehra, R., \& Singh, M., 2012. Estimation of radiological risk due to concentration of ${ }^{238} \mathrm{U},{ }^{226} \mathrm{Ra},{ }^{232} \mathrm{Th}$ and ${ }^{40} \mathrm{a} \mathrm{K}$ in soils of different geological origins in northern India. Turkish Journal of Physics, 36(2), 289-297.

Munyao, L. N., Ketui, D. K., Otieno, C., \& Chege, M. W., 2020. Assessment of levels of natural radioactivity in sand samples collected from Ekalakala in Machakos County, Kenya. The Scientific World Journal, 2020.

Najam, L. A., \& Younis, S. A., 2015. Assessment of natural radioactivity level in soil samples for selected regions in Nineveh Province (Iraq). International Journal of Novel Research in Physics Chemistry \& Mathematics, 2(2), 1-9.

Saleh, H., \& Shayeb, M. A., 2014. Natural radioactivity distribution of southern part of Jordan (Ma' an) Soil. Annals of Nuclear Energy, 65, 184-189.

UNSCEAR, 2000. Sources and effects of ionizing radiation. Sources, 1

UNSCEAR, 2014. Sources and effects of ionizing radiation. Report to the General Assembly, 2008, New York, United Nation.

Veiga, R., Sanches, N., Anjos, R. M., Macario, K., Bastos, J., Iguatemy, M., ... \& Umisedo, N. K., 2006. Measurement of natural radioactivity in Brazilian beach sands. Radiation measurements, 41(2), 189-196. 